\title{
Multiscale Edge Detection via Normal Changes ${ }^{\star}$
}

\author{
Chwen-Jye Sze ${ }^{1}$, Hong-Yaun Mark Liao ${ }^{2}$, Hai-Lung Hung ${ }^{3}$, Kuo-Chin Fan ${ }^{1}$, \\ and Jun-Wei Hsieh ${ }^{4}$ \\ ${ }^{1}$ Institute of Computer Science and Information Engineering, National Central \\ University, Chung-Li, Taiwan \\ 2 Institute of Information Science, Academia Sinica, Taiwan \\ ${ }^{3}$ Department of Electrical Engineering and Computer Science, Northwestern \\ University, IL 60208 , U.S.A. \\ 4 Computer and Communication Research Labs, Institute of Technology Research \\ Industry, Taiwan
}

\begin{abstract}
A new edge detection technique based on detection of normal changes is proposed. Most of the existing range image-based edge detection algorithms base their detection criterion on depth or curvature changes. However, the depth change-based approach does not have keen sensitivity in detecting roof (or crease) edges, and the curvature change-based approach suffers from a complicated and tedious principal curvature derivation process. Using normal changes as a detecting criterion, on the other hand, the existence of an edge can be easily detected, even when the change across a boundary is slight. Experimental results using both synthetic and real images demonstrate that the proposed method can efficiently detect both step and roof edges.
\end{abstract}

\section{Introduction}

In this paper, we propose a new edge detection technique based on detection of normal changes. The normal value is an important characteristic in differential geometry[10]. We find that by detecting normal changes, both step edges and roof edges can be easily identified. The whole detection procedure is divided into two stages. In the first stage, the normal of every point in a range image is decided. Since all the data points in a range image are discrete, the partial derivatives which are required to derive the normal value cannot be directly computed. For comparison purposes, we propose use of quadratic surface fitting[11], the orthogonal wavelet-based approach $[1,12]$, and the non-orthogonal wavelet-based approach[2], respectively, to approximate the original object surfaces and to then calculate the normal value of every discrete point on the surfaces. After the normal values of all the surface points are determined, the non-orthogonal wavelet transform ( dyadic wavelet transform ) proposed by Mallat et al. [2,13] is applied to detect those points which have significant normal changes as edge

* This work is supported by the National Science Council of Taiwan under grant no. NSC86-2745-E-001-004. 
points. From the experimental results, we find that the non-orthogonal waveletbased approach can best approximate the original surfaces from a discrete data set. Further, we also find that edge detection based on normal changes is a more promising alternative than other methods that base their detection criterion on depth or curvature changes. The proposed edge detector can detect both step edges and roof edges without introducing any edge models or heuristics.

\section{Range edge detection via normal change}

In this section, we shall explain why normal change can be used as a cue for range edge detection. Some properties of a 3D surface from the differential geometry viewpoint which are useful for edge detection will be addressed in Section 2.1. Then, a detailed explanation of why normal change is a better choice for edge detection will be given in Section 2.2 .

\subsection{Some properties in differential geometry useful for edge detection}

In this section, some basic properties of a 3D surface will be addressed from the differential geometry viewpoint. These properties are useful for solving the edge detection problem on range images.

Let $\mathcal{S}$ be a differentiable surface and $p(u, v)$ be a point on $\mathcal{S}$ with coordinate $(u, v)$. If $\boldsymbol{p}_{u}$ and $\boldsymbol{p}_{v}$ are the partial derivatives of $\boldsymbol{p}(u, v)$ with respect to $u$ and $v$, respectively, then we can say that $\boldsymbol{p}_{u}, \boldsymbol{p}_{v}$ form the basis of a tangent plane, $T(p)$, of $\boldsymbol{p}(u, v)$ (Figure 1). The normal of $T(p)$ can be defined as

$$
\boldsymbol{N}(u, v)=\frac{\boldsymbol{p}_{u} \times \boldsymbol{p}_{v}}{\left\|\boldsymbol{p}_{u} \times \boldsymbol{p}_{v}\right\|}
$$

Here, the norm of any $N(u, v)$ is always equal to 1 , and all $N(u, v)^{\prime} s$ lie on a
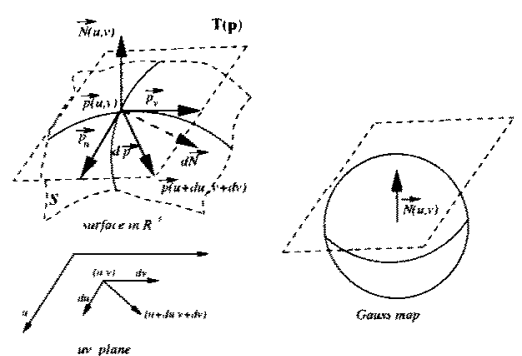

Fig. 1. Relations between a surface point, its corresponding parameter coordinate and the Gauss map.

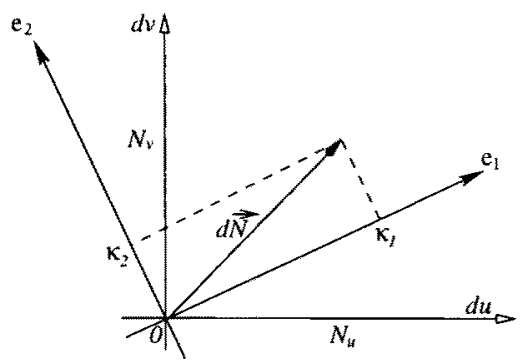

Fig. 2. Relationship between $d N$ and principal curvatures. 
unit sphere in $R^{3}$. The mapping, $N: \mathcal{S} \rightarrow R^{3}$, is called the Gauss mapping, $G\left(R^{3}\right)[10,14]$.

Let $N(u, v)$ be differentiable; the mapping, $d N(u, v)$, is from $G\left(R^{3}\right)$ to a tangent plane, $G\left(R^{3}\right)$, at $N(u, v)$. Since the tangent plane of $N(u, v)$ is equal to that of $\boldsymbol{p}(u, v), d \boldsymbol{N}(u, v)$ is also on the $T(p)$ plane, as shown in Figure 1. Thus, both $d p$ and $d N$ can be represented by the linear combination of $d u$ and $d v$ as follows $[10,7]$ :

$$
d \boldsymbol{p}=\boldsymbol{p}_{u} d u+\boldsymbol{p}_{v} d v
$$

and

$$
d N=N_{u} d u+N_{v} d v .
$$

Here, $p(u, v)$ is the gray level (intensity image) or depth (range image) at position $(u, v)$. Therefore, $d p$ physically means the intensity or depth change with respect to $\boldsymbol{p}(u, v)^{\prime} s$ neighbors. As for $\boldsymbol{N}_{u}$ and $\boldsymbol{N}_{v}$, they are mathematically defined as $\boldsymbol{N}_{u}=\frac{\partial \boldsymbol{N}}{\partial u}$ and $\boldsymbol{N}_{v}=\frac{\partial \boldsymbol{N}}{\partial u}$. That is, they physically mean the normal change along the $u$ and $v$ directions, respectively.

\subsection{Why normal change is better for edge detection}

Comparing the normal change-based approach and the curvature-based approach $[4,6]$, the computational complexity of the former is much less than that of the latter. As shown in Figure 2, $\{d u, d v\}$ and $\left\{e_{1}, e_{2}\right\}$ are two independent orthonormal bases on the tangent plane of $\boldsymbol{p}$. Based on differential geometry, the two principal curvatures, $\kappa_{1}$ and $\kappa_{2}$, can be derived by projecting $d N$ on a specific basis. Let $\left\{e_{1}, e_{2}\right\}$ be the basis with $e_{1}$ and $e_{2}$ corresponding, respectively, to the directions of the two principal curvatures, $\kappa_{1}$ and $\kappa_{2}[10]$. From Figure 2, it is obvious that $d N$ can be represented by a linear combination based on either $\{d u, d v\}$ or $\left\{e_{1}, e_{2}\right\}$. One thing to be noted is that no matter how $d N$ is represented, its magnitude is independent of the basis selected. The only thing that will vary with respect to the basis change is the orientation of $d N$. Therefore, using the normal change directly to locate edge positions does not require that the values of two principal curvatures be explicitely determined. This fact explains why $d N$ can replace the two principal curvatures in edge detection and why using $d N$ is much more efficient than using $\kappa_{1}$ and $\kappa_{2}$.

\section{Calculating normals from discrete surface points}

In the previous section, we have discussed how the change of normals at every point on a surface (Equation (3)) can be used to detect edges. The continuous domain normal value derivation process is summarized as follows. Let $\boldsymbol{p}(u, v)=$ $(u, v, f(u, v))$ be a point located on a surface. The partial derivatives of $\boldsymbol{p}(u, v)$ in the $u$ and $v$ directions are

$$
\boldsymbol{p}_{u}=\frac{\partial \boldsymbol{p}(u, v)}{\partial u}=\left(\begin{array}{lll}
1 & 0 & f_{u}(u, v)
\end{array}\right)
$$


and

$$
\boldsymbol{p}_{v}=\frac{\partial \boldsymbol{p}(u, v)}{\partial v}=\left(\begin{array}{lll}
0 & 1 & f_{v}(u, v)
\end{array}\right) .
$$

Plugging these two values into Equation (1), the value of $N(u, v)$ can be rewritten as

$$
\begin{aligned}
N(u, v) & =\left(\frac{-f_{u}}{\sqrt{1+f_{u}^{2}+f_{v}^{2}}} \frac{-f_{v}}{\sqrt{1+f_{u}^{2}+f_{v}^{2}}} \frac{1}{\sqrt{1+f_{u}^{2}+f_{v}^{2}}}\right) \\
& =\left(n_{1}(u, v) n_{2}(u, v) n_{3}(u, v)\right),
\end{aligned}
$$

where $f_{u}=\frac{\partial f(u, v)}{\partial u}$ and $f_{v}=\frac{\partial f(u, v)}{\partial v}, n_{1}(u, v)=\frac{-f_{u}}{\sqrt{1+f_{u}^{2}+f_{v}^{2}}}, n_{2}(u, v)=\frac{-f_{v}}{\sqrt{1+f_{u}^{2}+f_{u}^{2}}}$ and $n_{3}(u, v)=\frac{1}{\sqrt{1+f_{u}^{2}+f_{v}^{2}}}$. The ranges of $n_{1}, n_{2}$ and $n_{3}$ are all bounded by $(-1,1)$. In real implementation, since all the data points acquired in a range image are discrete by nature, the above calculation does not apply. Therefore, we have to find an appropriate method to deal with this problem. In the implemenation stage, we split the edge detection procedure into two steps. In the first step, the normal at every point on a surface should be determined. This step involves calculation of partial derivatives on a set of discrete data points. Then, in the second step, a detector is required to accurately detect the points where significant variations of normals are encountered. In order to calculate the normals on a set of discrete surface points, some existing methods $[2,11,15,16]$ can be applied. For comparison purposes, we choose quadratic surface fitting $[7,11]$, the orthogonal wavelet-based approach[17], and the non-orthogonal wavelet-based approach[2] to calculate the normal value of every point on a surface.

\section{Detecting edges based on normal changes}

In $[2,18,13]$, Mallat and his students developed some pioneering works for multiscale edge detection based on gray level changes. Here, we shall review part of their work which will be useful in our work. Define two wavelet functions, $\psi^{1}(x, y)$ and $\psi^{2}(x, y)[2,18]$, where

$$
\psi^{1}(x, y)=\frac{\partial \theta(x, y)}{\partial x},
$$

and

$$
\psi^{2}(x, y)=\frac{\partial \theta(x, y)}{\partial y}
$$

$\theta(x, y)$ is a smoothing function whose integration over the full domain is equal to 1 and converges to 0 at infinity. These two functions have to satisfy the following conditions:

$$
\int_{-\infty}^{\infty} \int_{-\infty}^{\infty} \psi^{1}(x, y) d x d y=0
$$

and

$$
\int_{-\infty}^{\infty} \int_{-\infty}^{\infty} \psi^{2}(x, y) d x d y=0
$$


Let $f(x, y) \in \mathbf{L}^{2}(\mathbf{R})$. The so-called dyadic wavelet transform $[2,18]$ of $f(x, y)$ at scale $2^{j}$ along the $x$ and $y$ directions can be represented, respectively, by

$$
W_{2^{j}}^{1} f(x, y)=f * \psi_{2^{j}}^{1}(x, y),
$$

and

$$
W_{2^{j}}^{2} f(x, y)=f * \psi_{2^{j}}^{2}(x, y),
$$

where $\psi_{2^{j}}^{1}(x, y)=\frac{1}{2^{2 j}} \psi^{1}\left(\frac{x}{2^{j}}, \frac{y}{2^{j}}\right)$ and $\psi_{2^{j}}^{2}(x, y)=\frac{1}{2^{2 j}} \psi^{2}\left(\frac{x}{2^{j}}, \frac{y}{2^{j}}\right)$. In what follows, we shall use the above mentioned dyadic wavelet transform to detect significant normal changes as edge points.

From Equation(3), it is obvious that the vector of the normal change, $d N(u, v)$, can be represented by the linear combination of the two bases on the $d u-d v$ plane, i.e., $N_{u} d u+N_{v} d v$. Also, their associated weights are the gradients of $N$ along the $d u$ and $d v$ directions, respectively. Since the dyadic wavelet transform proposed by Mallat $[2,13]$ can be used to calculate the magnitudes of these gradients, we can apply their method directly to calculate $d N(u, v)$. According to the formulation reported in $[2,13]$, the vector dyadic wavelet transform of $N(u, v)$ at scale $2^{j}$ can be defined as follows:

$$
\mathcal{W}_{j} N(u, v)=W_{2^{j}}^{1} N(u, v) d u+W_{2^{j}}^{2} N(u, v) d v,
$$

where

$$
W_{2^{j}}^{1} N(u, v)=\left(W_{2^{j}}^{1} n_{1}(u, v), W_{2^{j}}^{1} n_{2}(u, v), W_{2^{j}}^{1} n_{3}(u, v)\right)
$$

and

$$
W_{2^{j}}^{2} N(u, v)=\left(W_{2^{j}}^{2} n_{1}(u, v), W_{2^{j}}^{2} n_{2}(u, v), W_{2^{j}}^{2} n_{3}(u, v)\right) .
$$

Since the $\mathcal{W}_{j} N(u, v)$ vector also lies on the $d u$-dv plane, the magnitude and argument of $\mathcal{W}_{j} N(u, v)$ can be directly computed. Referring to [2], the norms of $W_{2^{j}}^{1} N(u, v)$ and $W_{2^{j}}^{2} N(u, v)$ should be defined, respectively, as follows:

$$
\left\|W_{2^{j}}^{1} N(u, v)\right\|=\sqrt{\left[W_{2^{j}}^{1} n_{1}(u, v)\right]^{2}+\left[W_{2^{j}}^{1} n_{2}(u, v)\right]^{2}+\left[W_{2^{j}}^{1} n_{3}(u, v)\right]^{2}},
$$

and

$$
\left\|W_{2^{j}}^{2} N(u, v)\right\|=\sqrt{\left[W_{2^{j}}^{2} n_{1}(u, v)\right]^{2}+\left[W_{2^{j}}^{2} n_{2}(u, v)\right]^{2}+\left[W_{2^{j}}^{2} n_{3}(u, v)\right]^{2}} .
$$

The magnitude of $\mathcal{W}_{j} N(u, v)$ at scale $2^{j}$ can, thus, be computed as follows:

$$
M_{2^{j}} N(u, v)=\sqrt{\left[\left\|W_{2^{j}}^{1} N(u, v)\right\|\right]^{2}+\left[\left\|W_{2^{j}}^{2} N(u, v)\right\|\right]^{2}} .
$$

Furthermore, the angle of $\mathcal{W}_{j} N(u, v)$ with respect to $d u$ direction is

$$
A_{2^{j}} N(u, v)=\operatorname{argument}\left(\left\|W_{2^{j}}^{1} N(u, v)\right\|+i\left\|W_{2^{j}}^{2} N(u, v)\right\|\right) .
$$

From the above calculations, every point in a range image will obtain two values. One is the magnitude of its normal change with respect to its neighbors, and the other is the direction tendency of this point. Like other multiscale edge detection methods $[2,18,19]$, the edge points can be determined by locating those local extrema whose normal changes exceed a preset threshold. 


\section{Experimental results}

In the experiments, a number of synthetic and real range images were adopted as test images to corroborate the effectiveness of the proposed method. Figure 3 showed a synthetic image : agpart. The size of the agpart image was $240 \times 240$. We also used two real images with different sizes. In the first stage of the experiment, the normal of every point in a range image had to be decided. In order to make a comparison, we used three different methods, i.e., quadradic surface fitting, and orthogonal and non-orthogonal wavelet-based approaches, to calculate the normal values. For this part we used the synthetic images, "agpart", as the test image. The experimental results of this part are shown in Figure 4. Figures 5(a)(c) show, respectively, the multiscale edges $\left(2^{1}, 2^{2}\right.$, and $\left.2^{3}\right)$ detected from three differently approximated surfaces. Among them, It is apparent that, when the quadratic surface fitting or the orthogonal wavelet-based approach was adopted to estimate the normal values, the detected edges contained some spurious results or the original edges delocalized from their original position. On the other hand, the edges detected from the normals as estimated by the non-orthogonal waveletbased approach were the best results. One thing to be noted is that the first stage of the proposed approach, i.e., the normal determination step, is crucial because a "poor" estimation of normal values may have an irrecoverable effect on the edge detection stage. A poor estimation method may smooth out the original image and, thus, delocalize edges from their correct locations. After comparing the empirical results, the non-orthogonal wavelet-based approximation was chosen because it produced the best results out of the three methods. Figures 6 and 7 show the results of a sequence of experiments based on real range images. All of these results were obtained by applying the non-orthogonal wavelet-based approach to estimate the normal values. From the results, we can find that most of the crease ( roof) edges were detected correctly.

\section{Conclusion and discussion}

In this paper, we have proposed a new edge detection technique based on detection of normal changes. We have found that, by detecting normal changes, both step edges and roof edges can be easily identified. Therefore, the new technique has been proven to be a more promising method than other methods that base their detection criterion on depth or curvature changes.

\section{References}

1. J. W. Hsieh, M. T. Ko, H. Y. Mark Liao, and K. C. Fan, "A new wavelet-based edge detector via constrained optimization", Image and Vision Computing, 1997, to appear.

2. S. Mallat and S. Zhong, "Characterization of signal from multi-scale edges", IEEE Transactions on Pattern Analysis and Machine Intelligence, vol. 14, no. 7, pp. 710-732, July 1992. 


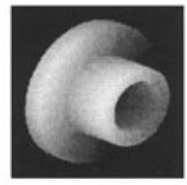

Fig. 3. A synthetic range image: agpart.

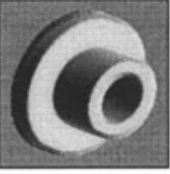

$m(u, n)$

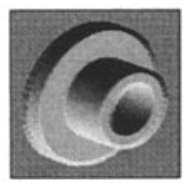

afa,n

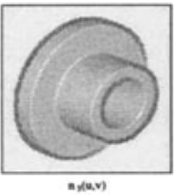

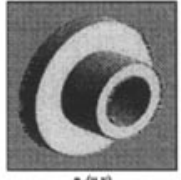

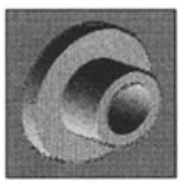

Au,

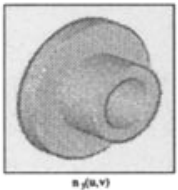

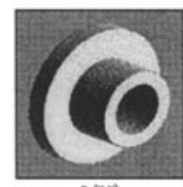

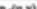

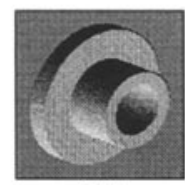

anda,n

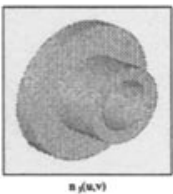

Fig. 4. Three component of normals $\left(n_{1}, n_{2}, n_{3}\right)$ estimated by using (a) quadratic surface fitting (b) the orthogonal wavelet-based approach with vanishing moment $\mathrm{N}=3$, and (c) the nonorthogonal wavelet-based approach.

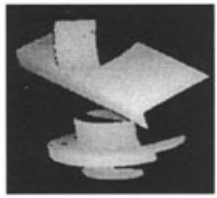

(w) Orymal nese inese

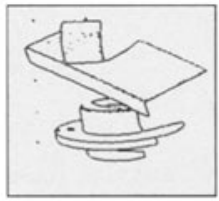

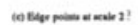

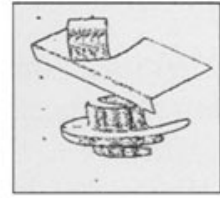

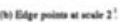

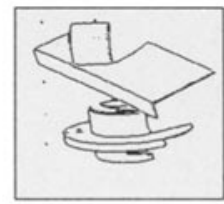

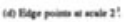

Fig. 6. Detected edge points at different scales from real image "occl".

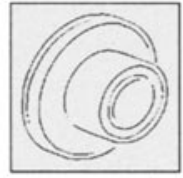

Eaye pints at kole 2 !

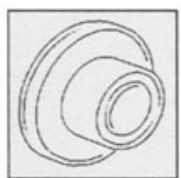

Fage pints a kale 2?

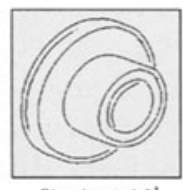

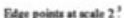

(*)

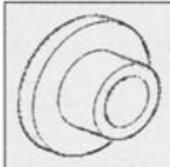

Bdye poinc at wale 2 !

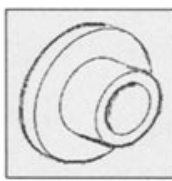

Edese pieter st kcale $2^{2}$

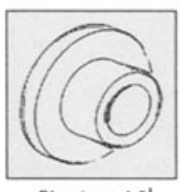

(a)

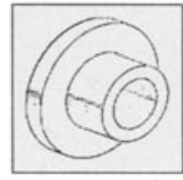

EAye piast at wale 2!

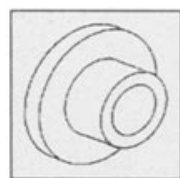

EAge pian at wate 2:

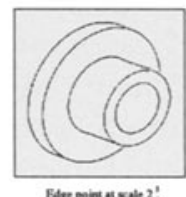

Fig. 5. Detected edge points at different scales using (a) quadratic surface fitting (b) the orthogonal wavelet-based approach with vanishing moment $\mathrm{N}=3$, and (c) the non-orthogonal waveletbased approach.

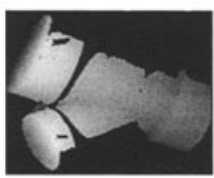

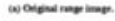

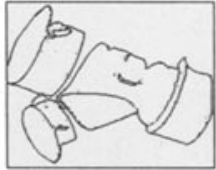

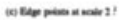

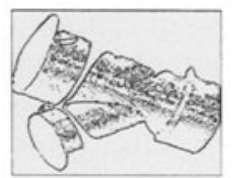

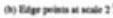

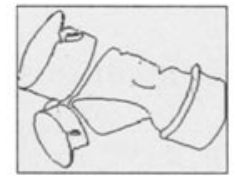

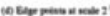

Fig. 7. Detected edge points at different scales from real image "bigwye". 
3. S. Ghosal and R. Mehrotra, "Segmentation of range images: an orthogonal moment-based integrated approach", IEEE Transactions on Robotics and Automation, vol. 61, no. 2, pp. 171-189, Aug. 1993.

4. O. Monga and S. Benayoun, "Using partial derivatives of $3 \mathrm{D}$ images to extract typical surface features", Computer Vision and Image Understanding, vol. 61, no. 2, pp. 171-189, Mar. 1995.

5. O. Monga, R. Deriche, G. Malandain, and J. P. Cocquerez, "Recusive filtering and edge tracking: two primary tools for 3D edge detection", Image and Vision Computing, vol. 9, no. 4, pp. 203-214, Aug. 1991.

6. T. J. Fan, G. Medioni, and R. Nevatia, "Segmented descriptions of 3-D surfaces", IEEE Transactions on Robotics and Automation, vol. 3 , no. 6, pp. 527-538, Dec. 1987.

7. P. J. Besl, Surface in range image understanding, Spring-Verlag, New York, 1988.

8. H. M. Cung, P. Cohen, and P. Boulanger, "Multiscale edge detection and classification in range images", in IEEE International Conference on Robotics and Automation, 1990 , pp. 2038-2044.

9. M. Djebali, M. Melkemi, and D. Vandorpe, "3D range image segmentation based on Deriche's optimun filters", in Proceedings of IEEE International Conference on Image Processing, 1994, pp. 503-507.

10. M. P. Do. Carmo, Differential Geometry of Curves and Surfaces, Prentice-Hall, Englewood Cliffs, NJ, 1976.

11. P. J. Besl and R. C. Jain, "Invariant surface characteristics for three-dimensional object recognition in range images", CVGIP, vol. 33, pp. 33-80, 1986.

12. I. Daubechies, "The wavelet transform, time-frequency localization and signal analysis", IEEE Trans. Information Theory, vol. 36, no. 5, pp. 961-1005, Sept. 1990.

13. S. Mallat, "Multiresolution approximation and wavelet orthonormal bases of $l\left(r^{2}\right)$ ", Transactions on Amer. Math., vol. 15, no. 3, pp. 69-87, Sept. 1989.

14. J. McCleary, Geometry from a Differentialable Viewpoint, Cambridge university, Cambridge, 1994.

15. I. Daubechies, "Orthonormal bases of compactly supported wavelets", Communications Pure Applied Mathematics, vol. 41, pp. 909-996, 1988.

16. X. Zhou R. O. Wells, "Wavelet interpolation and approximate solutions of elliptic partial differential equations", Tech. Rep., Computational Mathematics Laboratory, Rice University, 1993.

17. J. W. Hsieh, H. Y. Mark Liao, M. T. Ko, and K. C. Fan, "Wavelet-based shape from shading", Graphical Models and Image Processing, vol. 57, no. 4, pp. 343-362, July 1995.

18. S. Mallat and W. L. Hwang, "Singularity detection and processing with wavelets", IEEE Transactions on Information Theory, vol. 38, no. 2, pp. 617-643, March 1992.

19. J. W. Hsieh, H. Y. Mark Liao, M. T. Ko, K. C. Fan, and Y. P. Hung, "Image registration using an edge-based approach", Computer Vision and Image Understanding, 1997, to appear. 